\title{
Percepção dos enfermeiros sobre os acadêmicos de enfermagem acerca da sua inserção nos
} estágios

Nurses' perception regarding internship insertion of nursing students

\section{Percepción de los enfermeros sobre de los académicos de enfermería acerca de su inserción en las pasantías}

\section{Recebido: 21/06/2018 Aprovado: 15/01/2019 Publicado: 13/05/2019}

Jurema Ribeiro Luiz Gonçalves ${ }^{1}$ Álvaro da Silva Santos ${ }^{2}$

Leila Aparecida Kauchakje Pedrosa ${ }^{3}$ Camila Cristina Neves Romanato ${ }^{4}$ Maysa Alvarenga Ferreira ${ }^{5}$ Nara dos Santos Costa Daniela Sarreta Ignacio ${ }^{7}$ Mariana Mila Guimarães ${ }^{8}$ Judete Silva Nunes ${ }^{9}$

Ryanne Agnes Souza Silva de Oliveira ${ }^{10}$ Fabiano Henrique Oliveira Sabino ${ }^{11}$ Fernanda Lemos Lazarini12 Bruna Claúdia Pansani Ferreira ${ }^{13}$ Julia Cristina Molina Silveira ${ }^{14}$ Ketry Kellen Prado Caetano ${ }^{15}$

Esse trabalho teve como objetivo conhecer a percepção dos enfermeiros da atenção primária, acerca da inserção dos acadêmicos de graduação em enfermagem nos campos de prática. Trata-se de um estudo qualitativo realizado a partir de questões chaves, com 25 enfermeiros da rede de atenção primária e secundária do Município de Uberaba-MG, em setembro de 2017, com análise através do Discurso do Sujeito Coletivo. Emergiram duas categorias e cinco Discursos do Sujeito Coletivo. Foram reconhecidos pontos positivos e negativos sob a inserção do acadêmico no campo de prática na atenção primária. 0 acadêmico foi visto como agente de mudança e colaborador no trabalho de equipe e na assistência, sendo os pontos críticos a baixa valorização da atenção primária, a reduzida participação dos enfermeiros no planejamento das atividades e o despreparo e pouco interesse por alguns estudantes. Os enfermeiros percebem a inserção dos acadêmicos de modo favorável, entretanto consideram deficitária a comunicação e integração entre universidade e serviço.

Descritores: Atenção primária à saúde; Estudantes de enfermagem; Estágios.

This study aimed to know the perception of nurses in primary care about the integration of nursing undergraduate students in practice areas. This is a qualitative study, carried out from key issues, with 25 nurses from primary and secondary network of the city of Uberaba-MG, Brazil, in September 2017, with analysis of Discourse of the Collective Subject. Two categories and five discourses of the collective subject emerged. Positive and negative aspects on the integration of the academic in the practice field in primary care were recognized. One saw the academic as a change agent and collaborator on teamwork and care, being the critical points the low valuation of primary care, the reduced participation of nurses in the activities planning and the lack of preparation and little concern by some students. Nurses realize the integration of the students favorably; however, they consider communication and integration deficient between university and service.

Descriptors: Primary Health Care; Students, Nursing; Traineeships.

Ese trabajo tuvó como objetivo conocer la percepción de los enfermeros de la atención primaria, acerca de la inserción de los académicos de graduación en enfermería en los campos de prácticas. Se trata de un estudio cualitativo realizado a partir de cuestiones claves, con 25 enfermeros de la red de atención primaria y secundaria del Municipio de Uberaba , MG, Brasil, en septiembre de 2017, con análisis a través del Discurso del Sujeto Colectivo. Emergieron dos categorías y cinco Discursos del Sujeto Colectivo. Fueron reconocidos puntos positivos y negativos bajo la inserción del académico en el campo de prácticas en la atención primaria. El académico fue visto como agente de cambio y colaborador en el trabajo de equipo en la asistencia, siendo los puntos críticos la baja valoración de la atención primaria, la reducida participación de los enfermeros en la planificación de las actividades y a la falta de preparación y poco interés por algunos estudiantes. Los enfermeros perciben la inserción de los académicos de modos favorable, sin embargo consideran deficitaria la comunicación e integración entre universidad y servicio.

Descriptores: Atención primaria de salud; Estudiantes de enfermería; Pasantías.

1. Enfermeira. Mestre e Doutora em Enfermagem Psiquiátrica. Professora do Programa de Pós-Graduação em Atenção à Saúde da Universidade Federal do Triângulo Mineiro (UFTM). Uberaba, MG, Brasil. ORCID: 0000-0003-4448-8063 E-mail: juremaluiz@hotmail.com.br

2. Enfermeiro. Especialista em Saúde Pública. Mestre em Administração em Serviços de Saúde. Doutor em Ciências Sociais. Pós Doutor em Serviço Social. Professor Associado nos Programas de Pós-Graduação em: Atenção à Saúde e Psicologia da UFTM, Uberaba, MG, Brasil. ORCID 0000-0002-8698-5650 E-mail: alvaroenf@hotmail.com

3. Enfermeira. Mestre e Doutora em Enfermagem. Professora do Programa de Pós-Graduação em Atenção à Saúde da UFTM. Uberaba, MG, Brasil. ORCID: 0000-0003-1924-6142 E-mail: leila.kauchakje@enfermagem.uftm.edu.br

4. Enfermeira. Mestre em Atenção à Saúde. Doutoranda em Atenção à Saúde pela UFTM, Uberaba, MG, Brasil. ORCID: 0000-0001-6361-7292 E-mail: camilaromanatoribeiro@gmail.com

5. Enfermeira. Mestre em Atenção à Saúde. Doutoranda em Atenção à Saúde pela UFTM, Uberaba, MG, Brasil. ORCID: 000-0002-4483-0693 E-mail: maysalvarenga@gmail.com

6. Enfermeira. Mestre em Atenção à Saúde. Professora Substituta pela UFTM, Uberaba, MG, Brasil. ORCID: 0000-0002-6980-6147 E-mail: narasc29@gmail.com

7. Enfermeira. Mestre em Enfermagem Fundamental. Coordenadora e docente da FAFRAM, Ituverava, SP, Brasil. ORCID: 0000-0002-1432-5098 E-mail: daniela.sarreta@gmail.com

8. Enfermeira. Especialista em Saúde da Família. Uberaba, MG, Brasil. ORCID: 0000-0002-9459-5687 E-mail: marianamilaguimaraes@hotmail.com

9. Enfermeira. Odontóloga, Mestranda em Atenção à Saúde, pela UFTM, Uberaba, MG, Brasil. ORCID: 0000-0002-9455-9731 E-mail: judetenunes@uol.com.br

9. Enfermeira. Odontóloga, Mestranda em Atenção à Saúde, pela UFTM, Uberaba, MG, Brasil. ORCID: 0000-0002-9455-9731 E-mail: j

10. Acadêmica de Enfermagem pela UFTM, Uberaba, MG, Brasil. ORCID: 0000-0002-4171-323X E-mail: ryanne_agnes@hotmail.com
11. Acadêmico de Enfermagem pela UFTM, Uberaba, MG, Brasil. ORCID: 0000-0001-8728-9238 E-mail: fabianooliveira163@gmail.com

11. Acadêmico de Enfermagem pela UFTM, Uberaba, MG, Brasil. ORCID: 0000-0001-8728-9238 E-mail: fabianooliveira163@gmail.com

12. Acadêmica de Enfermagem pela UFTM, Uberaba, MG, Brasil. ORCID: 0000-0003-3688-3952 E-mail: fernandalaz@outlook.com
13. Acadêmica de Enfermagem pela UFTM, Uberaba, MG, Brasil. ORCID: 0000-0003-1764-7348 E-mail: bruna.pansani@hotmail.com

14. Acadêmica de Enfermagem pela UFTM, Uberaba, MG, Brasil. ORCID: 0000-0002-2970-1199 E-mail: juliaa-molina@ @otmail.com

15. Enfermeira. Biomédica. Uberaba, MG, Brasil. ORCID: 0000-0003-4448-8063 E-mail: ketrykellen@ hotmail.com 


\section{INTRODUÇÃO}

A Atenção Primária é caracterizada por intervenções com o intuito de promoção, proteção e reabilitação da saúde no âmbito individual e coletivo ${ }^{1}$. De forma integral e continua às práticas dentro das unidades básicas fortalecem vínculos com usuários/ comunidade e cria um rico campo de aprendizagem e troca de experiências para acadêmicos e profissionais ${ }^{2}$.

Entre a equipe multiprofissional que compõe uma Unidade Básica de Saúde (UBS), os enfermeiros são os que mantêm maior contato com os usuários dos serviços, pela diversidade de atividades que desempenha e por coordenar as atividades dos Agentes Comunitários de Saúde (ACS) e dos profissionais da enfermagem. Além de suas contribuições diárias como visitas domiciliares, serviços administrativos e assistenciais, em muitas unidades o enfermeiro atua como preceptor na formação de graduandos do curso de Enfermagem, em visitas técnicas, ensino clínico e no estágio curricular obrigatório, entre outros ${ }^{3}$.

Considera-se também que nas atividades práticas nos serviços de saúde, o enfermeiro preceptor, apesar de não pertencer à instituição ocupa um importante papel e complementa conceitos e práticas ainda não vivenciados durante a graduação ${ }^{4}$.

0 estágio curricular supervisionado (ECS) é o espaço de oportunidade triangulada da realidade profissional concreta listada pela vivência, interação teórico-prática e enfrentamento do conhecimento, possibilizando a reflexão e paralelismo a respeito do curso e das categorias destinadas à formação. Dessa forma, compreende-se que os diferentes ambientes de estágio, ou seja, instituições de saúde e a academia tendem se unirem cada vez mais, proporcionando para o mercado de trabalho profissionais capazes para enfrentar os obstáculos e também oferecer reflexões acerca dos objetivos e metas no processo de formação do aluno e como a instituição de ensino enxerga e discute esse assunto em suas demandas diárias ${ }^{5}$.

Nessa reflexão a prática em campo é vista pela enfermagem como o espaço para construção do profissional, além da reflexão da prática assistencial, bem como o amadurecimento e oportunidade de interação do acadêmico com a realidade do seu futuro trabalho. Entretanto, por vezes essa inserção ocorre de modo tardio e não baseada na autonomia de aprendizado do aluno ${ }^{5}$.

Tendo em vista, os avanços necessários para inserção precoce do acadêmico na rede de atenção à saúde, faz-se necessário conhecer a percepção dos agentes envolvidos no processo.

O objetivo desse trabalho foi conhecer a percepção dos enfermeiros da atenção primária, acerca da inserção dos acadêmicos de graduação em enfermagem nos campos de prática.

\section{METODO}

Trata-se de um estudo qualitativo realizado durante ao mês de setembro de 2017 com enfermeiros da rede de atenção primária e secundária da Secretaria Municipal de Saúde (SMS) de Uberaba-MG. O contato com os sujeitos da pesquisa foi realizado por e-mail, no qual foi apresentado a proposta da pesquisa.

A coleta de dados foi realizada por meio de entrevistas individuais coletadas nas modalidades de formulário online via e-mail e impresso, conforme a escolha do entrevistado.

Foi elaborado um roteiro semiestruturado, composto por dados de caracterização do participante e três questões abertas que versavam sobre idade, tempo de formado, distrito sanitário de saúde em que trabalhava e, tempo de trabalho na SMS.

Fizeram parte do roteiro os seguintes questionamentos: Qual a sua percepção sobre os acadêmicos de Enfermagem, durante o contato com a rede? Por quê? Como você vê a interação dos acadêmicos de Enfermagem da UFTM com os serviços de saúde? Quais sugestões você teria para aprimorar a formação dos acadêmicos do curso de Enfermagem da UFTM, de acordo com a necessidade do serviço de saúde (rede municipal)?

Os dados numéricos foram analisados segundo estatística descritiva, para caracterização do perfil dos participantes. 
Para a análise das respostas abertas, utilizouse o Discurso do Sujeito Coletivo (DSC) ${ }^{6,7}$.

O DSC é uma técnica de processamento de depoimentos, em que os discursos são agrupados segundo o significado do conteúdo e similaridade, dando sentido durante a narração da coletividade e representatividade do discurso de um determinado grupo, criado, por meio, das representações sociais que emergem de maior quantidade de (expressõeschave $)^{6,7}$.

Esta pesquisa é uma parte de um estudo maior, intitulado "Descortinando os meandros da formação em enfermagem: percepções construídas no contexto da universidade e serviços de saúde", que foi aprovado pelo Comitê de Ética em Pesquisa da Universidade Federal do Triângulo Mineiro, sob o número de CAE n².590.76. Para a realização do estudo foram considerados os princípios éticos estabelecidos pela Resolução 466/12.

\section{RESULTADOS}

Dos 51 enfermeiros que compõe a SMSUberaba, participaram 25 destes. Os sujeito dessa pesquisa, em sua maior parte, possuíam 7 a 10 anos de experiência profissional na área da Enfermagem e 35 a 44 anos de idade. A Tabela 1 apresenta a distribuição dos participantes deste estudo, segundo os dados sociodemográficos.

Tabela 1. Distribuição sociodemográficas dos enfermeiros da Secretaria Municipal de Saúde. Uberaba, Setembro de 2017.

\begin{tabular}{llc}
\hline Variável & \multicolumn{2}{l}{ Frequência } \\
\hline Idade & $\mathrm{N}$ & $(\%)$ \\
\hline $25-34$ anos & 11 & $(44)$ \\
$35-44$ anos & 13 & $(52)$ \\
$45-50$ anos & 01 & $(04)$ \\
\hline Tempo de formação & & \\
\hline $1-6$ anos & 08 & $(32)$ \\
$7-10 \quad$ nos & 13 & $(52)$ \\
$\geq 17$ anos & 04 & $(16)$ \\
\hline Tempo de experiência & & \\
\hline$<1$ ano & 04 & $(16)$ \\
$1|-| 10$ anos & 11 & $(44)$ \\
$11|-| 15$ anos & 09 & $(36)$ \\
$\geq 16$ anos & 01 & $(04)$ \\
\hline Total & 25 & $(100)$ \\
\hline
\end{tabular}

A partir de uma primeira leitura dos relatos, foram extraídas as expressões-chave e as ideias centrais, sendo selecionadas aquelas que fossem semelhantes para construção dos discursos. Posteriormente, categorizaram-se e classificaram-se os depoimentos emergindo duas categorias, que contribuem para a melhor compreensão dos resultados:

\section{Categoria 1. Aspectos positivos da inserção do acadêmico de enfermagem no serviço}

As atividades práticas na atenção básica e especializada são vistas de forma positiva pelos enfermeiros, no que se refere às pesquisas e diagnóstico situacionais desenvolvidas em campo, pois a partir delas, torna-se possível traçar um melhor planejamento das ações de enfermagem, conforme apresentado no discurso 1:
DSC 1: Considero (muito) boa a interação (positiva), pois vivenciam toda a prática e desafios do enfermeiro na saúde pública, considerando o trabalho em equipe $e$ liderança, mesmo que em alguns locais seja somente no âmbito da pesquisa. Ajudam a detectar quais os problemas mais frequentes na rede de atenção à saúde do município, favorecendo que nossos gestores tracem melhores estratégias e que, nós enfermeiros, melhoremos nossa assistência, aprimorando-a a todo instante através de novos conhecimentos.

A presença dos acadêmicos de Enfermagem na rede é percebida pelos enfermeiros como algo positivo, pois colaboram para a melhoria da qualidade da assistência, no exercício do trabalho em equipe e liderança, como no DSC 2:

DSC 2: Muito importante, positivo/bom; são de fundamental importância para o serviço na saúde pública, acrescentam ao serviço e, contribuem no desenvolvimento das atividades executadas na unidade. 
Segundo os enfermeiros, o contato com os serviços de saúde propicia ao aluno oportunidade de vivenciar de modo prático o conteúdo teórico, contribuindo na sua formação. Em relação ao perfil destes acadêmicos sob a ótica dos enfermeiros, eles são interessados e responsáveis, proporcionam vínculo com a equipe e a população, tornando o serviço menos árduo, como no DSC 3:

DSC 3: Os acadêmicos são pró-ativos, muito colaborativos, dedicados, comprometidos e responsáveis, interessados em compreender como a rede funciona. Os estudantes se beneficiam ao adquirir novos conhecimentos e responsabilidades, amadurecem e preparam-se para intervir na sociedade e a partir deste contato é possível adequar o conteúdo teórico com a prática.

\section{Categoria 2. Aspectos negativos da inserção do acadêmico de enfermagem no serviço}

No entanto, os entrevistados pontuaram alguns aspectos negativos dessa inserção como o conhecimento deficitário do funcionamento da rede, das atribuições do profissional enfermeiro, dos conteúdos e técnicas básicas para a atuação na Atenção Primária a Saúde (APS), para que haja um melhor aproveitamento das atividades práticas, como no DSC 4:

DSC 4: Não é passado, aos enfermeiros da rede, o que eles têm que vivenciar na prática daquela disciplina, naquele momento do estágio. A carga horária é bem pequena, $e$ ainda não dão a devida importância à atenção básica; alguns apenas desempenham o papel de observação. Percebe-se que ainda não tem maturidade suficiente para compreender a importância do funcionamento da rede, para o desempenho de suas funções enquanto profissional, são despreparados, sem conhecimento sobre o SUS e seu funcionamento. O pouco conhecimento em relação aos fluxos de atendimento existentes no município, também apresentam dificuldades em aprender determinadas técnicas ou procedimentos. Porém ao longo dos estágios e com a maior compreensão do sistema, os acadêmicos conseguem interagir $e$ integrar à rede de saúde.

Os profissionais acreditam que a rede municipal é pouco explorada pela universidade. Citam como ponto crítico a não participação dos profissionais e serviços no planejamento e funcionamento das práticas acadêmicas, sendo elencadas no discurso a seguir:

DSC 5: Na maioria das vezes não há um contato pelos professores, anteriormente, para traçar os objetivos do estágio. Necessitam maior envolvimento e engajamento nos programas de atenção básica da rede. Falta um maior contato entre professor-coordenador e enfermeiro preceptor para definir as reais atribuições para os acadêmicos; é necessário mostrar a realidade de todos os tipos de serviços de saúde.

\section{DISCUSSÃO}

Em pesquisa na qual foi foram entrevistados 45 enfermeiros que já atuaram como supervisores de estágio hospitalar, a inserção no campo de práticas também foi vista como uma oportunidade de construção do conhecimento teórico associado à prática, em um espaço que o ocorre tanto o crescimento acadêmico quanto profissional, dos acadêmicos, enfermeiros e da equipe ${ }^{8}$.

Em outro estudo com narrativas de estudantes de enfermagem de universidade pública sobre vivências no estágio na área de atenção básica e hospitalar, verificou-se contribuição do estágio para a formação profissional, por afirmarem ser o cenário ideal para a apropriação do trabalho, sendo ainda, o enfermeiro percebido como agente chave na organização da equipe e do cuidado ${ }^{9}$.

Assim como descrito neste estudo, outra investigação descreve como pontos críticos e dificuldades enfrentadas pelos enfermeiros preceptores, o despreparo e imaturidade dos acadêmicos ${ }^{8}$. Entretanto, é esperado que enquanto acadêmicos, os estagiários tenham sentimentos e atitudes de insegurança que estão relacionados ao processo normal de aprendizagem e amadurecimento. Ao mesmo tempo outros estudos expressaram alternância do acadêmico, entre se perceber como mão de obra de trabalho e em outros momentos como aprendiz ${ }^{9}$

Em relação a integração ensino e serviço é recorrente a opinião tanto em estudos com enfermeiros quanto em estudos com discente e docentes, a necessidade de valorização, participação no planejamento das atividades em campo de estágio ${ }^{8}$. Aspecto levantado em estudo semelhante sob a perspectiva de estagiários, destacou-se o papel do docente enquanto articulador entre instituição de ensino e serviço, promovendo a comunicação e aproximação do enfermeiro com o processo de ensino aprendizagem ${ }^{10}$.

A relação entre docência-assistência pode proporcionar um maior e amplo 
conhecimento das atividades de enfermagem. Porém, a articulação entre o ensino e o serviço podem gerar conflitos, o que prejudica essa articulação. Os preceptores se sentem desamparados e muitas das vezes desatualizados sobre o plano de ensino e a real necessidade do estágio para o acadêmico. Em estudo com acadêmicos e docentes, foi destacado a necessidade de que profissionais preceptores e supervisores sejam capacitados para trabalhar de modo conjunto as demandas individuais dos acadêmicos, tendo destaque a figura do enfermeiro como referência enquanto profissional ${ }^{11}$.

A aproximação entre preceptores e professores representa um passo fundamental na construção de estratégias para trocas mais produtivas no dia a dia dos estágios e assim, uma divisão mais justa e igualitária de responsabilidades entre a universidade e os serviços de saúde na formação dos enfermeiros ${ }^{12}$.

0 enfermeiro preceptor é uma das bases para um ensino de qualidade e implementação das políticas públicas de saúde. É imprescindível que sejam investigados fatores que contribuem para a manutenção da desarticulação entre ensino e prática, o que pode oferecer subsídios para que sejam desenvolvidas estratégias voltadas à mudança de caráter satisfatório e qualidade tanto para profissionais, acadêmicos e usurários da APS 13 .

Pode-se perceber que há divergências entre o ensino e a prática de enfermagem. A universidade, por seguir padrões tradicionais, por vezes, deixa de explorar campos da APS e foca somente na atenção terciária, deixando um déficit no conteúdo e práticas para o acadêmico neste campo.

Apesar do déficit de conteúdo o acadêmico contribui de forma positiva para os profissionais compartilhando novas práticas e atualidades na área. Porém, a falta de integralização e o distanciamento com o docente responsável caracteriza um dos nós críticos mais preocupantes pelos preceptores. Assim como relatado pelos enfermeiros, os acadêmicos também fazem críticas referentes a desvalorização da APS e valorização do modelo hospitalocêntrico ${ }^{9}$.
Cabe ao enfermeiro preceptor compartilhar experiências e técnicas satisfatórias e de qualidade que ajudem o graduando se adaptar à futura prática profissional. Além de instruir, aconselhar e ensinar deve possuir uma boa comunicação, com vistas a propiciar um ambiente de confiança para os graduandos, em atividades diárias e enfatizar que o ambiente de trabalho passa por constantes mudanças e é necessário se adaptar a elas.

Existe uma diferença entre estágio e trabalho, pois o trabalho requer do profissional trabalhador decidir a atividade que desenvolverá, já no estágio o aluno tem o professor para supervisionar e orientar sobre as decisões das atividades, sendo que o docente interfere sobre elas. Portanto, faz-se necessário uma profunda discussão entre trabalho e educação por meio das legislações dos estágios, bem como, das políticas públicas que possam garantir ao acadêmico direito efetivo para sua formação o aproximando do seu campo de estágio ${ }^{5}$.

\section{CONCLUSÃO}

Os enfermeiros que recebem estudantes no campo de estágio, acreditam que a docênciaassistência melhora e amplia o conhecimento dos acadêmicos, bem como a assistência da enfermagem, porém ressalta a geração de conflitos que há entre serviço e ensino em alguns momentos e isso prejudica tanto os acadêmicos, quanto a enfermagem assistencial, a docência e os usuários, pois a falta de comunicação diminui o vínculo que sobre percepção dos profissionais é o ponto chave para uma boa aprendizagem e assistência.

Os preceptores enfatizaram a necessidade de estarem atualizados com o plano de ensino, bem como necessitam da melhora na comunicação entre docente e preceptor para que quando receberem os acadêmicos nos campos de estágio saberão orientar de forma mais concisa e pontual as dúvidas e as necessidades do acadêmico sem prejudicar a assistência. Ou ainda, melhorar a assistência justamente pelo fato do sistema ter novos olhares para os problemas antigos, sendo assim, melhora da qualidade da 
assistência e ainda sujem novas ideias para resolução de conflitos já existentes, de forma justa e igualitária para os acadêmicos, professores, preceptores e usuários.

As limitações são relativas ao estudo ter sido aplicado somente a nível local e pela baixa resposta ao convite de participação na pesquisa.

Pesquisas nesta área de conhecimento podem colaborar para a melhoria da articulação entre a universidade e os profissionais do campo de prática. Bem como, contribuir na melhoria da qualidade do curso de enfermagem.

\section{REFERÊNCIAS}

1. Ministério da Saúde (Br). Política nacional de atenção básica. Brasília, DF: Ministério da Saúde; 2012. 114p.

2. Ministério da Saúde (Br), Secretaria de Atenção à Saúde, Departamento de Atenção Básica. Por uma cultura da paz, a promoção da saúde e a prevenção da violência. Brasília, DF: Ministério da Saúde; 2009. 48p.

3. Tavares PEN, Santos SAM, Comassetto I, Santos RM, Santana VVRS. A vivência do ser enfermeiro e preceptor em um hospital escola: olhar fenomenológico. Rev Rene [Internet]. 2011 [citado em 03 maio 2018]; 12(4):798-807. Disponível em: http://periodicos.ufc.br/rene/article/view/4 344

4. Botti SHO, Rego S. Preceptor, supervisor, tutor e mentor: quais são seus papéis? Rev Bras Educ Med. [Internet]. 2008 [citado em 03 maio 2018]; 32(3):363-73. Disponível em: http://www.scielo.br/pdf/rbem/v32n3/v32 n3a11.pdf

5. Marran AL, Lima PG, Bagnato MHS. As políticas educacionais e o estágio curricular supervisionado no curso de graduação em enfermagem. Trab Educ Saúde [Internet]. 2015 [citado em 03 maio 2018]; 13(1): 89$108 . \quad$ Disponível em: http://www.scielo.br/pdf/tes/v13n1/1981-7746tes-1981-7746-sip00025.pdf

6. Lefevre F, Lefevre AMC. Pesquisa de representação social: um enfoque qualitativo: a metodologia do discurso do sujeito coletivo. 2ed. Brasília, DF: Liberlivro Editora; 2012.
7. Lefevre F, Lefevre AMC. Discurso do sujeito coletivo: representações sociais e intervenções comunicativas. Texto \& Contexto Enferm. 2015; 23(2):502-7.

8. Souza DJ, Faria MF, Cardoso RJ, Contim D. Estágio curricular supervisionado sob a óptica dos enfermeiros supervisores. Rev Enferm Atenção Saúde [Internet]. 2017 [citado em 03 maio 2018]; 6(1):39-51. Disponível em: http://seer.uftm.edu.br/revistaeletronica/in dex.php/enfer/article/view/1677

9. Rossi NF, Fortuna CM, Matumoto S, Marciano FM, Silva JB, Silva JS. As narrativas de estudantes de enfermagem nos portfólios do Estágio Curricular Supervisionado. Rev Eletrônica Enferm. [Internet]. 2014; [citado em 03 maio 2018]; 16(3):566-74. Disponível em: http://revistas.ufg.br/index.php/fen/art icle/view/25691

10. Marchioro D, Ceratto PC, Bitencourt JVOV, Martini JG, Silva Filho CC, Silva TG. Estágio curricular supervisionado: relato dos desafios encontrados pelos (as) estudantes. Arq Ciênc Saúde UNIPAR. [Internet]. 2017; [citado em 03 maio 2018]; 21(2):119-22. Disponível em: http://www.revistas.unipar.br/index.ph $\mathrm{p} /$ saude/article/view/5912. DOI: https://doi.org/10.25110/arqsaude.v21i2.20 17.5912

11. Rigobello JL, Bernardes A, Moura AA, Zanetti ACB, Spiri WC, Gabriel CS. Estágio curricular supervisionado e o desenvolvimento das competências gerenciais: a visão de egressos, graduandos e docentes. Esc Anna Nery Rev Enferm. [Internet]. 2018 [citado em 03 maio 2018]; 22(2):e20170298. Disponível em: http://www.scielo.br/pdf/ean/v22n2/pt_14 14-8145-ean-2177-9465-EAN-2017-

0298.pdf.

DOI: http://dx.doi.org/10.1590/2177-9465-ean2017-0298

12. Padilha MICS. Análise crítica das causas de integração e/ou desintegração docente assistencial na enfermagem. Rev Gaúch Enferm. 1991; 12(1):33-7.

13. Trevisan D, Minzon DT, Testi CV, Ramos NA, Carmona, EV, Silva EM. Formação de enfermeiros: distanciamento entre a graduação e a prática profissional. Ciênc Cuid Saúde [Internet]. 2013[citado em 03 maio 
2018]; 12(2):331-7. Disponível em: http://www.periodicos.uem.br/ojs/index.ph p/CiencCuidSaude/article/viewFile/19643/ pdf

CONTRIBUIÇÕES
Jurema Ribeiro Luiz Gonçalves, Álvaro
da Silva Santos e Leila Aparecida e
Kauchakje Pedrosa foram responsáveis
pelo desenho do estudo, coleta de dados,
análise dos dados, correção do manuscrito e
orientação das etapas da pesquisa. Camila
Cristina Neves Romanato, Maysa
Alvarenga Ferreira e Nara dos Santos
Costa realizaram análise dos dados,
fundamentação teórica, normalização
bibliográfica e redação. Daniela Sarreta
Ignacio, Mariana Mila Guimarães e
Judete Silva Nunes contribuíram na coleta
de dados, levantamento bibliográfico e
revisão. Ryanne Agnes Souza Silva de
Oliveira, Fabiano Henrique Oliveira
Sabino, Fernanda Lemos Lazarini, Bruna
Claúdia Ransani Ferreira, Julia Cristina
Molina Silveira e Ketry Kellen Prado
Caetano foram responsáveis pela coleta de
dados e revisão.

CONTRIBUIÇÕES

Jurema Ribeiro Luiz Gonçalves, Álvaro da Silva Santos e Leila Aparecida e Kauchakje Pedrosa foram responsáveis pelo desenho do estudo, coleta de dados, análise dos dados, correção do manuscrito e orientação das etapas da pesquisa. Camila Cristina Neves Romanato, Maysa Alvarenga Ferreira e Nara dos Santos Costa realizaram análise dos dados, fundamentação teórica, normalização bibliográfica e redação. Daniela Sarreta Ignacio, Mariana Mila Guimarães e de dados, levantamento bibliográfico e revisão. Ryanne Agnes Souza Silva de Oliveira, Fabiano Henrique Oliveira Sabino, Fernanda Lemos Lazarini, Bruna Claúdia Ransani Ferreira, Julia Cristina Caetano foram responsáveis pela coleta de dados e revisão.

\section{Como citar este artigo (Vancouver)}

Gonçalves JRL, Santos AS, Pedrosa LAK, Romanato CCN, Ferreira MA, Costa NS, et al. Percepção dos enfermeiros sobre os acadêmicos de enfermagem acerca da sua inserção nos estágios. REFACS [Internet]. 2019 [citado em inserir dia, mês e ano de acesso]; 7(2):220-226. Disponível em: inserir link de acesso. DOI: inserir link do DOI.

\section{Como citar este artigo (ABNT)}

GONÇALVES, J. R. L.; SANTOS, A. S.; PEDROSA, L. A. K.; ROMANATO, C. C. N.; FERREIRA, M. A.; COSTA, N. S. et al. Percepção dos enfermeiros sobre os acadêmicos de enfermagem acerca da sua inserção nos estágios. REFACS, Uberaba, MG, v. 7, n. 2, p. 220-226, 2019. DOI: inserir link do DOI. Disponível em: inserir link de acesso. Acesso em: inserir dia, mês e ano de acesso.

\section{Como citar este artigo (APA)}

Gonçalves, J.R.L., Santos, A.S., Pedrosa, L.A.K., Romanato, C.C.N., Ferreira, M.A., Costa, N.S., et al. (2019). Percepção dos enfermeiros sobre os acadêmicos de enfermagem acerca da sua inserção nos estágios. REFACS, 7(2), 220-226. Recuperado em: inserir dia, mês e ano de acesso. Inserir link de acesso. DOI: inserir link do DOI. 